\title{
OBSTRUCCIÓN INTESTINALE INTUSUSCEPCIÓN POR PÓLIPO FIBROIDE INFLAMATORIO DE YEYUNO. INFORME DE CASO
}

INTESTINAL OBSTRUCTION AND INTUSSUCEPTION BY A FIBROID INFLAMATORY POLYP OF THE JEJUNUM. A CASE REPORT OBSTRUÇÃO INTESTINAL E INUSUSCEPÇÃO POR POLIPO FIBROIDE INFLAMATÓRIO DE YEYUNO. RELATÓRIO DE CASO

\author{
JAMES NEIRA BOR JA1,2, DIEGO PAREDES CALAHORRANO², LÚVER MACÍAS JARA², JHON CORONEL RUILOVA², FÁTIMA NEIRA \\ VERDUGA ${ }^{3}$ \\ Universidad de Guayaquil, Guayaquil, Ecuador. \\ 2 Hospital Luis Vernaza, Guayaquil, Ecuador \\ ${ }^{3}$ Omnihospital, Guayaquil, Ecuador.
}

Resumen

Introducción: el tracto gastrointestinal se constituye en un lugar común de presentación de tumores de diversa índole, pero se han descrito escasos casos de pólipos de naturaleza fibroide inflamatoria, que se acompañan de obstrucción intestinal. Se presenta el caso clínico de un paciente masculino de 52 años, con antecedentes de consumo de alcohol y un cuadro de dolor abdominal difuso de 2 meses de evolución que presentó un pólipo fibroide inflamatorio, benigno, y conjuntamente un cuadro de obstrucción intestinal, sujeto de resolución de tipo quirúrgico. Se realiza revisión bibliográfica del tema.

PALABRAS CLAVE: pólipos intestinales, intususcepción, diagnóstico, leiomioma, yeyuno.

Abstract

Introduction: the gastrointestinal tract is one of the most common places for the development of a wide variety of tumors. Few cases of intestinal obstruction caused by fibroid like polyps have been reported. Objetive: to present a clinical case of a benign inflammatory fibroid poylp that causes intestinal obstruction that needed a surgical treatment. A bibliografic review has been made of this topic. The clinical class of a 52 -year-old male patient is presented, with a history of alcohol consumption and a diffuse abdominal pain picture of 2 months of evolution that presented an inflammatory, benign fibroid polyp, and together a picture of intestinal obstruction, subject to Resolution of surgical type. A bibliographic review of the subject has been made

KEYWORDS: intestinal polyps, intussusception, diagnosis, leiomyoma, jejunum.

Resumo

INTRODUÇão: 0 trato gastrointestinal é um local comum para a apresentação de tumores de vários tipos, mas poucos casos de pólipos de natureza fibroide inflamatória foram descritos, os quais são acompanhados por obstrução intestinal. Objetivo: apresentar um caso clínico de pólipo fibro-inflamatório benigno que apresente quadro de obstrução intestinal, sujeito a resolução do tipo cirúrgico. Uma revisão bibliográfica do assunto foi feita. Apresenta-se a classe clínica de um paciente do sexo masculino, 52 anos, com histórico de consumo de álcool e quadro de dor abdominal difusa de 2 meses de evolução que apresentou pólipo fibromioide inflamatório benigno e, juntos, um quadro de obstrução intestinal, sujeito a Resolução do tipo cirúrgico. Foi feita uma revisão bibliográfica do assunto. PALAVRAS-CHAVE: pólipos intestinais, intussuscepção, diagnóstico, leiomioma, jejuno. 
INTRODUCCIÓN

La presentación de masas de tipo polipoide fibroide inflamatorio, en la población adulta, es algo poco frecuente. Descrito inicialmente por Vanek en 1949, a nivel mundial se han reportado aproximadamente 90 casos desde 1986. Constituyen lesiones granulomatosas inespecíficas, más comúnmente encontrados en población con edad comprendida entre los 50 a 80 años. ${ }^{1,2}$ Se describe clásicamente como una lesión que se presenta más frecuentemente en antro gástrico, yeyuno e ileon, infrecuente en el colon y excepcionalmente en el esófago, siendo su naturaleza benigna. . $^{3,4}$

Se manifiesta al ulcerarse con cuadros de anemia, obstrucción intestinal por intususcepción y rara vez síntomas sistémicos cuando afectan a estómago o yeyuno. ${ }^{6,7}$

Histológicamente es una lesión generalmente única que se encuentra en la zona alta de la submucosa o en la profundidad de la mucosa del tracto intestinal, constituido por células fusiformes y fibras conjuntivas dispuestas concéntricamente alrededor de elementos vasculares con un grado de infiltrado de tipo inflamatorio eosinofílico. ${ }^{8}$

Se considera que es una lesión expansiva y reactiva posiblemente a una agresión local múltiple, ${ }^{9}$ se han descrito como posibles desencadenantes a los traumas locales, la cirugía, los antiinflamatorios no esteroideos, agentes microbianos o la enfermedad de Crohn. ${ }^{10,11}$ Se clasifican dentro del grupo de tumores correspondientes a pólipos del intestino delgado, mesenquimatosos de linaje no determinado por completo, constituyendo apenas menos del $5 \%$ de los tumores gastrointestinales. ${ }^{12}$

Su sintomatología puede acompañarse por alteraciones de laboratorio, siendo la más llamativa la anemia, la relación de esta lesión con marcadores tumores alterados se ve demostrada por las características de la lesión y la expresión de dichos marcadores inmunohistoquímicos. ${ }^{13}$

Los pólipos fibroides inflamatorios, son de naturaleza benigna, siendo las complicaciones locales, las más comunes con repercusiones sistémicas posteriores; no se ha reportado agresividad y características invasoras en este tipo de patología. ${ }^{14}$

Por ser tumores mesenquimatosos benignos, la incidencia de recidivas es en extremo infrecuente tras su extirpación ya sea por vía endoscópica o por cirugía abierta, aunque el diagnóstico es a veces dificultoso sin su resección; pese a los avances en técnicas diagnósticas con imágenes, aun hoy en día su diagnóstico y tratamiento continúan siendo un reto. ${ }^{15,16,17}$

\section{CASO CLÍNICO}

Paciente de sexo masculino, de 52 años de edad, sin antecedentes patológicos de importancia, hábito alcohólico semanal, hasta la embriaguez; acude a emergencia, por cuadro clínico de 2 meses de evolución, caracterizado por dolor abdominal difuso, que se exacerba hace 24 horas, con dolor intenso, que no cede a analgesia proporcionada por familiares; el cuadro se acompaña de náusea que llega al vómito, abundante, por varias ocasiones, de contenido alimentario en inicio, y bilioso posterior; a la inspección el paciente luce pálido, sudoroso.

Al examen físico presenta constantes vitales: TA: 100/60, FC: 95 lat/min, FR: 20 rpm, FC: 95 lpm, SatO2: $99 \%$ sin oxígeno, T: $36^{\circ} \mathrm{C}$, Clasgow $15 / 15$; mucosas orales secas, abdomen: distendido, tenso, doloroso difuso, se observa y escucha movimientos peristálticos acentuados, se auscultan ruidos hidroaéreos disminuidos.

Los exámenes de laboratorio revelan: leucocitos 7740, neutrofilos 64.9\%, $\mathrm{Hb} 12.7$, Hto 38.9, plaquetas 308.000; amilasa $41 \mathrm{mg} / \mathrm{dl}$; Na 140 $\mathrm{mEq} / \mathrm{L}, \mathrm{K} 3.74 \mathrm{mEq} / \mathrm{L}, \mathrm{Cl} 100 \mathrm{mEq} / \mathrm{L}$; lipasa 23 $\mathrm{mg} / \mathrm{dL}$; PCR $64.05 \mathrm{mg} / \mathrm{dL}$; creatinina $0.85 \mathrm{mg} / \mathrm{dL}$; procalcitonina 0.08 .

En la placa de RX de abdomen, se visualiza patrón con intestino delgado muy dilatado muy probablemente a nivel de yeyuno con niveles hidroaéreos no llamativos puesto que se evacuó el contenido intestinal con sonda nasogástrica (Figura 1).

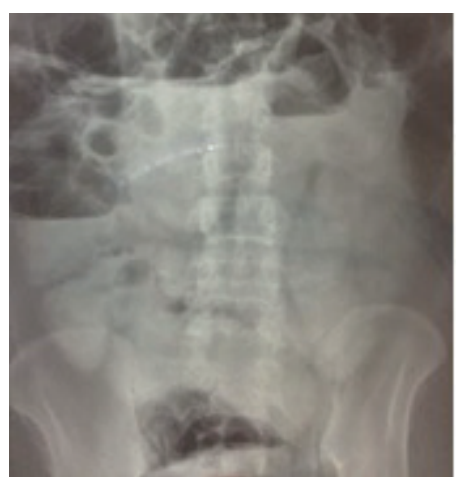

Figura 1. RX de abdomen que demuestra intestino delgado dilatado con presencia de niveles hidroaéreos.

Fuente: servicio de Radiodiagnóstico, hospital Luis Vernaza, 2015. 
La tomografía axial computarizada con contraste por vía oral, el mismo que llega hasta el colon sigmoides sin llegar a la ampolla rectal, y revela masa isodensa a nivel de intestino delgado (Figura $2,3)$.

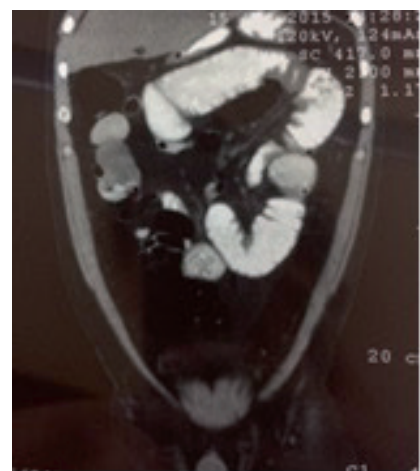

Figura 2. TAC contrastada de abdomen que demuestra el paso del contraste hasta el colon sigmoides, masa isodensa a nivel de intestino delgado. Fuente: servicio de Radiodiagnóstico, hospital Luis Vernaza, 2015.

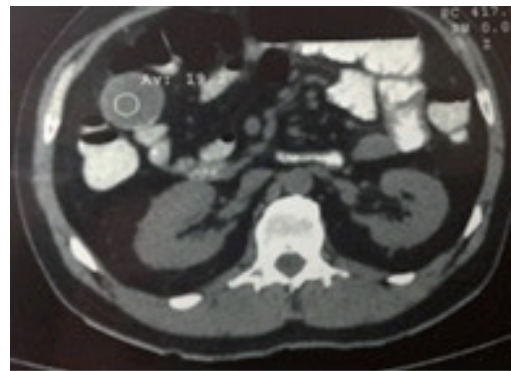

Figura 3. TAC contrastada de abdomen que demuestra el masa isodensa a nivel de intestino delgado.

Fuente: servicio de Radiodiagnóstico, hospital Luis Vernaza, 2015.

Posterior a estabilización de paciente, analgesia adecuada, valoraciones prequirúrgicas y complementación de exámenes de laboratorio e imagen; con un abdomen tenso, doloroso y distendido, se procede a realización de laparotomía exploratoria, encontrándose asas intestinales dilatadas, masa yeyunal de aproximadamente $8 \mathrm{~cm}$ de longitud con intususcepción a 2 metros del ángulo de Treitz (Figura 4).

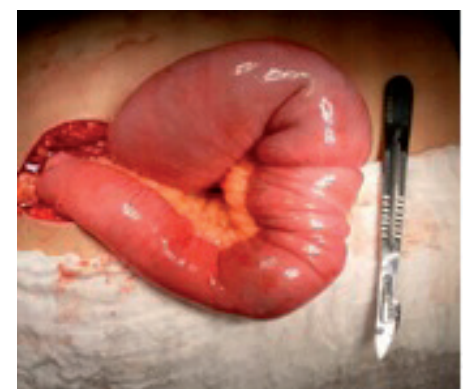

Figura 4. Masa yeyunal de aproximadamente $8 \mathrm{~cm}$, con intususcepción, aproximadamente a 2 metros del ángulo de Treitz.

Fuente: servicio de Cirugía General, hospital Luis Vernaza, 2015.
Con los hallazgos descritos se realiza resección de masa tumoral a $10 \mathrm{~cm}$ proximal y distal de sus márgenes, con muestra enviada a patología (Figura 5), se realiza anastomosis términoterminal, se limpia cavidad y se exteriorizan 2 drenajes desde corredera parietocólica izquierda y espacio rectovesical respectivamente; se cierra planos anatómicos. Cabe recalcar que no se observaron ni palparon ganglios linfáticos durante la intervención quirúrgica. Posteriormente el paciente pasa a sala de recuperación, en donde presenta taquipnea, control de gasometría arterial revela acidosis metabólica; se corrige estado metabólico y es enviado a hospitalización.

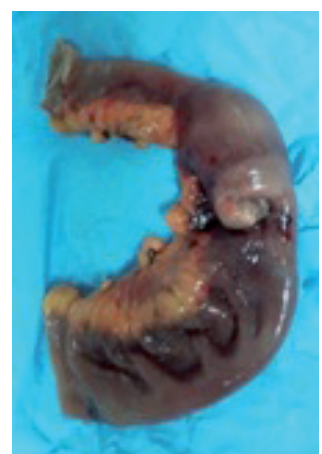

Figura 5. Masa yeyunal de aproximadamente $8 \mathrm{~cm}$, con intususcepción, aproximadamente a 2 metros del ángulo de Treitz, muestra enviada al servicio de patología.

Fuente: servicio de Cirugía General, hospital Luis Vernaza, 2015.

Durante su estancia el paciente presenta periodos de distensión abdominal, con eliminación de líquido intestinal por sonda nasogástrica, valorado por servicio de medicina interna, solicita perfil proteico que demuestra valores de: proteínas séricas totales $5.28 \mathrm{mg} / \mathrm{dL}$, seroalbúmina $2.86 \mathrm{mg} / \mathrm{dL}$, prealbúmina $4 \mathrm{mg} / \mathrm{dL}$; marcadores tumorales: Ca72.3: 0.8 U.I, Ca19.9: 0.37 U.I, alfafetoproteína: 0.66 U.I, antígeno carcinoembrionario: 0.54 U.I, Ca15.3: 6.7 U.I, Ca125: 8.5; se decide dar alimentación parenteral total.

Realiza pico febril durante su segundo día de hospitalización por lo que se rota antibiótico terapia, con control posterior adecuado, y drenajes con producción de líquido serohemático en constante disminución hasta su retiro definitivo al cuarto día posoperatorio, así como se retira sonda nasogástrica, con posterior avance a tolerancia oral de líquidos, pero por control de estado nutricional, continúa con nutrición parenteral, con evolución favorable, es dado de alta por parte de cirugía general, con tolerancia oral adecuada. 
Al octavo día posoperatorio se recibe resultado de examen de patología (Figura 6, 7).

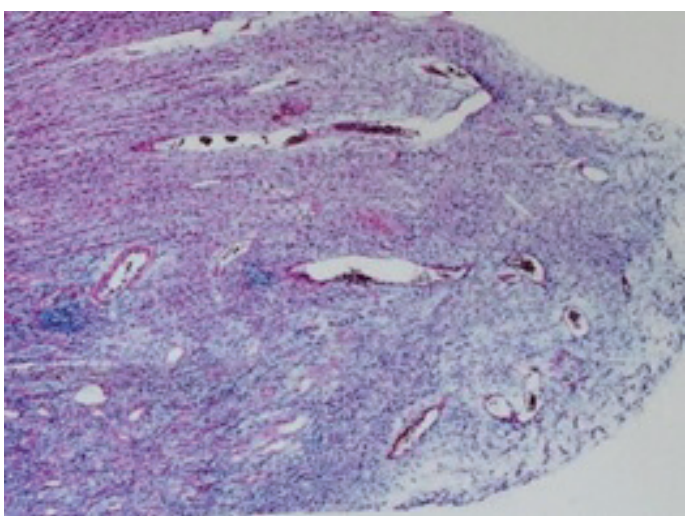

Figura 6. Pieza anatomopatológica que demuestra masa intraluminal con patrón característico de intususcepción y de aspecto benigno.

Fuente: servicio de Patología, Hospital Luis Vernaza, 2015.

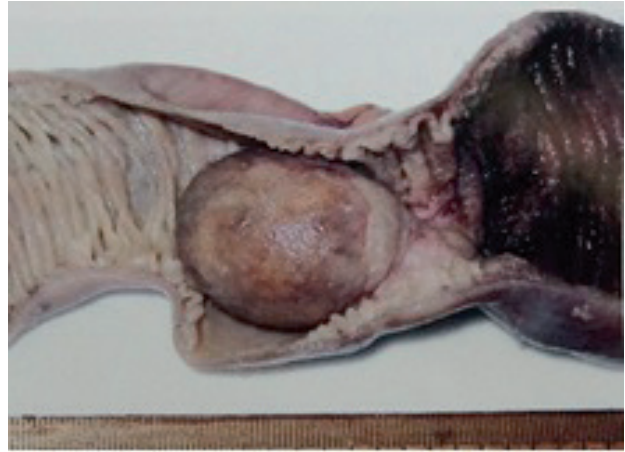

Figura 7. Corte microscópico que demuestra proliferación mesenquimatosa y fibroblástica con infiltrado de predominio eosinófilo, compatible con pólipo fibroide inflamatorio.

Fuente: servicio de Patología, hospital Luis Vernaza, 2015.

DISCUSIÓN

Los pólipos fibroides inflamatorios son lesiones sésiles o pedunculadas, de base inflamatoria inicialmente reportadas por Vanek en 1949. ${ }^{18}$ Helwig y Rainer propusieron el termino "pólipo fibroide inflamatorio" en 1953. ${ }^{19}$

Estas lesiones se encuentran en diversos grupos de edad, siendo más común su presentación entre la quinta a séptima décadas de la vida, puede situarse en cualquier parte del tracto gastrointestinal, siendo más comunes en el antro gástrico $(70 \%)$ y en yeyuno e ileon ( $25 \%)$. generalmente son asintomáticos detectados de manera incidental en exámenes de imagen; sin embargo cuando presentan sintomatología gastrointestinal, dependen de su localización anatómica; los síntomas obstructivos son los más comunes. Hobbs y Cohen en 1946, los describen por primera vez como causa importante de abdomen agudo inflamatorio, que generalmente se presentan con intususcepción. ${ }^{20}$
Histológicamente poseen características mesenquimatosas con predominio de fibroblastos e infiltrado de tipo eosinófilo.

En este caso, la clínica, conjuntamente con los hallazgos de los estudios de imagen, dieron lugar a la sospecha diagnóstica de la existencia de una masa intraluminal, potencialmente respetable conjuntamente con la porción intestinal afectada, debido a la ausencia de síntomas de tipo constitucional como caquexia y mal estado nutricional, además, no se hallaron imágenes sugestivas de compromiso linfático o invasión vascular.

Las características vistas mediante tomografía dieron lugar a la decisión de la resolución de un cuadro inflamatorio de tipo obstructivo. Otro auxiliar importante lo constituye el eco Doppler que puede detectar signos de neovascularización e invasión a estructuras vecinas; sin embargo, las imágenes obtenidas por tomografía axial computarizada, dieron la pauta para realizar el procedimiento quirúrgico. El análisis de los exámenes de laboratorio, conjuntamente con marcadores tumorales en valores no considerables, enmarcaron el manejo, a una resección del segmento intestinal afectado, anastómosis término-terminal en yeyuno, y manejo posquirúrgico con estabilización del estado general, que posteriormente llevaron a tomar la decisión de alta en espera del resultado de patología que 4 días después del procedimiento arrojaron los resultados comentados anteriormente. El seguimiento posterior del paciente es importante, sin embargo no se han descrito recidivas en este tipo de casos. La evolución final y los controles posoperatorios fueron adecuados.

\section{CONCLUSIONES}

Los pólipos fibroides inflamatorios, son entidades de presentación poco frecuente, son lesiones granulomatosas no específicas de etiología variada, que ocurren generalmente en adultos. Histologicamente se revela su origen de tipo inflamatorio, bacteriano, químico o traumático; puede ser una lesión sésil o polipoidea, que se desarrolla en la mucosa del tracto gastrointestinal; en este caso con presentación menos común de lo normal en yeyuno. La sintomatología es variada pero en estadios avanzados sobreviene la obstrucción intestinal con datos microscópicos de intususcepción. Ante esta sintomatología que demuestra un abdomen agudo inflamatorio, y con una adecuada valoración radiológica y del estado general del paciente, el tratamiento siempre será la resección 
de la lesión y su segmento intestinal comprometido. La bibliografía reporta que no existe potencial de malignidad en esta lesión.

DECLARACIÓN DE ORIGINALIDAD

Declaramos que el contenido del artículo es original y que no ha sido publicado como artículo previamente ni está enviado para ninguna consideración a cualquier otra publicación, en su totalidad o en alguna de sus partes.

\section{CONFLICTO DE INTERÉSES}

Los autores declaramos que no hay conflictos de intereses.

\section{REFERENCIAS BIBLIOGRÁFICAS}

1. Persoff M. Eosinophilic granuloma causing intussusception in a three year old child. Am J Surg. 1972; 124: 676-78

2. Kourie G. Reporte de un caso de pólipo fibroide inflamatorio del yeyuno. Instituto Dominicano de Se-guros Sociales, Santo Domingo, República Dominicana, 2002

3. Van de Rijn. An inmunohistochemical study of inflammatory fibroid polyp of the gastrointestinal tract. Appl Inmunohistochemical. 1994; 2:54-9

4. Shimer G. Inflammatory fibroid polyps of the intestine. Am J Clin Pathol. 1984;81:708-14

5. Wille P. Fibroid polyps of intestinal tract are inflammatoryreactive proliferations of CD34-positive perivascular cells. Histopathology. 1998;32:498-502

6. Molina M. Pólipo fibroide inflamatorio del esófago, fiebre y poliartritis. Gastroenterol Hepatol. 1989;12:66-7

7. Ordi J, Aligotas J, Villar M. Pólipo fibroide inflamatorio yeyunal como causa de síndrome febril. Gastroenterolo Hepatol. 1987;10:292-4
8. Navas-Palacios J, Colina-Ruizdelgado F. Inflammatory fibroid polyps of the gastrointestinal tract. An inmmunohistochemical and electron microscopy study. Cancer 1983;51:1682-90

9. Benjamin SP, Hawk WA. Fibrous inflammatory polyps of the ileum and cecum. Cancer. 1977;39:1300-5

10. Wu JP, Yui EJ. Inflammatory pesudotumor of the abdomen: plasma cell granulomas. J coin Pathol. 1973;26:943-8

11. Muñiz-Grijalbo O. Could a fibroid polyp be a manifestation of enteropathy induced by anti-inflammatory drugs. Am J Gastroenterol. 1997;92:170-1

12. Lappas JC. Benign tumors of the small bowel. Cap 44. En: Gore, Textbook of gastrointestinal radiol-ogy. 2da edición. WB Saunders; 2000, 785-91

13. Madrid de Haan P, Roa Y. Intusus-cepción intestinal debido a un pólipo fibroide inflamatorio (tumor de Vanek). Medicina Legal de Costa Rica. 2014;31

14. Schildhaus, HU. Inflammatory fi-broid polyps. Atlas Genet Cytogenet Oncol Haematol. 2013;17(9):647-649.

15. Carmack SW, Genta RM, Graham DY, Lauwers GY. Management of gastric polyps: a pathology based guide for gastroenterologists. Nat Rev Gastroenterol Hepatol. 2009 Jun;6(6):331-41

16. Pinto Pais T, Fernandes S. A large gastric inflammatory fibroid polyp. J Gastroenterol. Elsevier. 2015;22:61-4

17. MatsushitaM, Hajiro K. Okazaki K. Gastric inflammatory fibroid polyps: ultrasonographic analysis in comparison with the histology. Gastrointest Endosc. 1997;46:53-7

18. Vanek J. Gastric submucosal granu-loma with eosinophilic infiltration. Am J Pathol. 1949; 25:397-411.

19. Helwig EB, Ranier A. Inflammatory fibroid polyps. Surg Gynecol Obstet. 1953; 96:335-67.

20. Akbulut $S$. Intussusception due to inflammatory fibroid polyp: a case report and comprehensive literature review. World J Gastroenterol. 2012; 18:5745-52. 